\title{
EVALUATION OF PAIN ON INJECTION, HAEMODYNAMICS AND POSTOPERATIVE NAUSEA AND VOMITING FOLLOWING INDUCTION WITH PROPOFOL, ETOMIDATE-LIPURO AND COINDUCTION WITH PROPOFOL + ETOMIDATE LIPURO- A COMPARATIVE STUDY
}

\author{
Kunal Sharma ${ }^{1}$, Nandita Mehta², Sunana Gupta ${ }^{3}$
}

${ }^{1}$ Senior Resident, Department of Anaesthesiology, GMC, Jammu.

2Professor and HOD, Department of Anaesthesiology, ASCOMS and H, Jammu.

${ }^{3}$ Associate Professor, Department of Anaesthesiology, ASCOMS and H, Jammu.

\begin{abstract}
BACKGROUND
ABSTRACT

Induction of general anaesthesia is the most important phase, which can be achieved by various inhalational and intravenous agents. An ideal inducing agent for general anaesthesia should have haemodynamic stability and minimal respiratory depression, rapid clearance and minimal side effects. Propofol is the most commonly used induction agent, as it has rapid onset and early recovery due to short half-life and rapid elimination from the blood circulation. Propofol is associated with pain[1] during induction, which is sometimes very distressing to patients and it can also cause haemodynamic instability.

The aim of this study was to compare the incidence of pain on injection, haemodynamic changes following induction and the incidence of PONV using etomidate-lipuro, propofol and coinduction with propofol and etomidate-lipuro.
\end{abstract}

\section{MATERIALS AND METHODS}

This is a randomised, controlled trial. A total of 90 patients with ASA I and II were randomly allocated into three groups, i.e. Group E, Group P and Group P + E. Group E patients received etomidate $0.3 \mathrm{mg} / \mathrm{kg}$, Group P patients received propofol $2 \mathrm{mg} / \mathrm{kg}$ and Group P + E received propofol $1 \mathrm{mg} / \mathrm{kg}+$ etomidate $0.2 \mathrm{mg} / \mathrm{kg}$ for induction of anaesthesia. The incidence of pain on injection was observed during the infusion of drugs, haemodynamic changes including heart rate, systolic blood pressure, diastolic blood pressure and mean blood pressure were accessed after induction and after intubation at various time intervals. The incidence of PONV was accessed for $24 \mathrm{hrs}$. postoperatively.

\section{RESULTS}

Comparing the three groups, it was seen that the incidence of pain on injection was maximum in propofol group followed by etomidate group and was reduced to a highly significant level in etomidate + propofol group $(\mathrm{p}<0.0001)$. The haemodynamic (systolic, diastolic and mean blood pressures, heart rate) changes were minimal in Propofol + Etomidate group. Etomidate group was associated with higher incidence of postoperative nausea and vomiting.

\section{CONCLUSION}

We concluded that coinduction with etomidate-lipuro and propofol resulted in lesser incidence of injection pain, minimal haemodynamic changes and lesser incidence of postoperative nausea and vomiting as compared to either drug given alone for induction of anaesthesia.

\section{KEYWORDS}

Propofol, Etomidate-Lipuro, Coinduction.

HOW TO CITE THIS ARTICLE: Sharma K, Mehta N, Gupta S. Evaluation of pain on injection, haemodynamics and postoperative nausea and vomiting following induction with propofol, etomidate-lipuro and coinduction with propofol + etomidate lipuro- a comparative study. J. Evolution Med. Dent. Sci. 2018;7(02):176-180, DOI: 10.14260/jemds/2018/39

\section{BACKGROUND}

Induction of general anaesthesia is the most important phase, which can be achieved by various inhalational and intravenous agents. An ideal inducing agent for general anaesthesia should have haemodynamic stability and minimal respiratory depression, rapid clearance and minimal side effects.

'Financial or Other Competing Interest': None.

Submission 17-11-2017, Peer Review 20-12-2017,

Acceptance 26-12-2017, Published 08-01-2018.

Corresponding Author:

Dr. Sunana Gupta,

H. No. 14, Indira Colony,

Camp Road, Talab Tillo,

Jammu-180002,

$J$ and $K$, India.

E-mail: sunaynaarnav@yahoo.com

DOI: $10.14260 /$ jemds $/ 2018 / 39$

\section{(c) $($ ) $९$}

Propofol is the most commonly used induction agent, as it has rapid onset and early recovery due to short half-life and rapid elimination from the blood circulation. Propofol is associated with pain[1] during induction, which is sometimes very distressing to patients and it can also cause haemodynamic instability.(2)

Etomidate is a carboxylated imidazole derived nonbarbiturate known for its haemodynamic stability during general anaesthesia. At typical anaesthetic induction doses, etomidate produces minimal blood pressure and heart rate changes even in patients with valvular or ischaemic heart disease. But myoclonus during induction and postoperative nausea and vomiting are the most frequent cited side-effects of etomidate.(3)

Coinduction is the concurrent administration of two or more drugs to facilitate induction of anaesthesia. Coinduction reduces the dose of induction agent and produces desired effects in more appropriate and balanced manner with fewer side effects than that can be observed by using single drug. (4) 
Etomidate-lipuro and propofol combination in the ratio of 1: 1 can be used for induction in general anaesthesia. Combination of etomidate with propofol would decrease the required dose of both the medications and also decreasing the side effects associated with individual drug. The combination can be used to provide the benefits of both the agents.

This present study was conducted to compare the incidence of pain on injection, haemodynamics, postoperative nausea and vomiting associated with coinduction with propofol etomidate or either drug used alone for induction of anaesthesia.

\section{MATERIALS AND METHODS}

This is a randomised, controlled trial. After obtaining approval from the Institutional Ethics Committee and written consent, 90 patients presenting for elective surgery, under general anaesthesia between December 2014 and November 2015 were enrolled for this study. Sample size is taken conveniently. Study design was single centre, prospective, randomised, controlled trial. Randomisation was done using computer-generated randomisation schedule.

\section{Inclusion Criteria}

1. American Society of Anaesthesiologist, Grade I or II.

2. Age between 18 - 65 years of either sex.

3. MPG I and II.

\section{Exclusion Criteria}

1. History of allergy to study drugs.

2. History of seizure disorder.

3. Presence of primary and secondary steroid deficiency or on steroid medications.

Every patient underwent pre-anaesthetic check-up one day prior to surgery that included a detailed history, complete general physical and systemic examination and relevant investigations. Patients were given tablet midazolam $7.5 \mathrm{mg}$ and tablet pantoprazole $40 \mathrm{mg}$ at bedtime on night prior to surgery and were kept fasting $8 \mathrm{~h}$ prior to surgery. Patients were randomly divided using a computer-generated randomisation schedule to compose three equal groups of 30 patients each-

- Group P - Propofol (2 mg/kg)

- $\quad$ Group E - Etomidate $(0.3 \mathrm{mg} / \mathrm{kg})$

- Group P + E - Propofol (1 mg/kg) + Etomidate $(0.2$ $\mathrm{mg} / \mathrm{kg}$ )

In the preoperative room, an IV line was secured using 18-G cannula and ringer lactate was started. Injection Ranitidine $50 \mathrm{mg}$ and Ondansetron $0.1 \mathrm{mg} / \mathrm{kg}$ was given in preoperative room. All baseline parameters i.e. heart rate, blood pressure (systolic, diastolic, mean arterial pressure) and oxygen saturation were recorded on arrival in the operating room. Continuous monitoring of heart rate, blood pressure and saturation were done at regular intervals.

In the operation theatre, all patients received Injection Fentanyl $1 \mathrm{mcg} / \mathrm{kg}$ followed by propofol $2 \mathrm{mg} / \mathrm{kg}$ in Group P, etomidate $0.3 \mathrm{mg} / \mathrm{kg}$ in Group $\mathrm{E}$ and etomidate $0.2 \mathrm{mg} / \mathrm{kg}+$ propofol $1 \mathrm{mg} / \mathrm{kg}$ in Group P + E.
Study drugs were prepared by the resident who was not involved in the study. The syringes were prefilled to contain $20 \mathrm{~mL}$ of the drug for blinding purpose (no visual difference could be detected between syringes). The person who was giving the drug was unaware regarding which drug is being given.

Neuromuscular blockade was achieved with Injection Rocuronium $0.6 \mathrm{mg} / \mathrm{kg}$ in all the 3 groups, which was given at the end of the study drug injection and cuffed endotracheal tube of appropriate size was inserted after adequate muscle relaxation was achieved.

Pain on injection due to the study drug was measured during induction using pain graded scale, which include the following responses-

- No complaint of pain during infusion was graded 0 .

- Verbal complaint of pain during infusion was graded 1.

- Withdrawal of arm during infusion was graded 2.

- $\quad$ Both verbal complaint and withdrawal was graded 3.

Continuous monitoring of heart rate, blood pressure (systolic, diastolic, MAP), ECG and oxygen saturation was done 1 min after the study drug, 1 min after intubation and then 3, 5, 10 mins after intubation and thereafter at every 10 mins till the end of surgery.

Anaesthesia was maintained with $\mathrm{N} 20-02$ in ratio of 66\%:33\% with isoflurane in varying concentration in all the groups. Rocuronium was used in dose of $0.15 \mathrm{mg} / \mathrm{kg}$ to maintain neuromuscular blockade as and when required.

Injection Diclofenac sodium $1.5 \mathrm{mg} / \mathrm{kg}$ was also given by slow intravenous infusion intraoperative before completion of surgery.

At the end of the surgery, neuromuscular blockade was reversed with Injection neostigmine $0.05 \mathrm{mg} / \mathrm{kg}$ and glycopyrrolate $0.01 \mathrm{mg} / \mathrm{kg}$ body weight intravenously.

Tracheal extubation was done after proper suctioning and when extubation criteria was met and the patient was shifted to postoperative recovery room.

Monitoring of PONV was done postoperatively using Verbal Rating Scale for next 24 hrs.

\section{For Nausea \\ 0 - No nausea, \\ 1 - Mild nausea, \\ 2 - Moderate nausea, \\ 3 - Severe nausea.}

\section{For Vomiting}

0 - No vomiting,

1 - 1 episode (Mild),

2 - 2 or 3 episodes (Moderate),

3 - More than 3 episodes (Severe).

\section{Statistical Analysis}

All statistical analysis was performed using statistical packages for social science version 19 (SPSS, Inc., Chicago, IL, USA). Demographic data was compared using chi-square test. Quantitative data was compared using unpaired t-test. Statistical significance was considered if $\mathrm{P}<0.05$. Based on the previous data and with the power of study at $80 \%$ (alpha $=0.05 \%$ ), a sample size of ninety (30 in each group) was considered adequate. 


\section{RESULTS}

There was no statistical difference among the three groups in terms of their Age and Sex, ASA, MPG and Weight status of the patients $(\mathrm{p}>0.05)$ [Table 1].

\begin{tabular}{|c|c|c|c|}
\hline & Group P & Group E & Group E+P \\
\hline Age (Years) & $45 \pm 9.37$ & $44.60 \pm 10.97$ & $48.00 \pm 10.21$ \\
\hline Sex & & & \\
M & $17(56.67)$ & $19(63.33)$ & $16(53.33)$ \\
F & $13(43.33)$ & $11(36.67)$ & $14(46.67)$ \\
\hline ASA & & & \\
I & $20(66.67)$ & $21(70.00)$ & $20(66.67)$ \\
II & $10(33.33)$ & $9(30.00)$ & $10(33.33)$ \\
\hline MPG & $16(53.33)$ & $12(40.00)$ & $17(56.67)$ \\
I & $14(46.67)$ & $18(60.00)$ & $13(43.33)$ \\
II & $60.37 \pm 9.79$ & $57.70 \pm 9.29$ & $62.80 \pm 12.49$ \\
\hline Weight & \multicolumn{4}{|c|}{ Table 1. Demographic Data } \\
\hline \multicolumn{4}{|c}{} \\
\hline
\end{tabular}

The comparison of pain on injection between group $\mathrm{P}$, Group E and Group P + E was highly significant ( $<<0.0001)$. During the infusion of drugs, the incidence was maximum with propofol $(100 \%)$ followed by etomidate (16.67\%), while there was no pain on injection $(0.00 \%)$ when the mixture of propofol + etomidate was used in combination (Fig. 1).

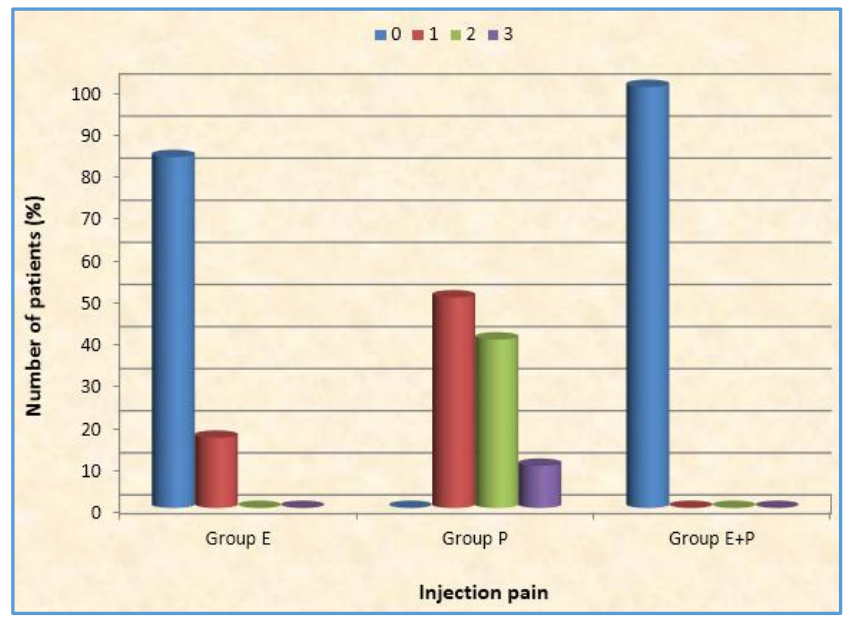

Figure 1

On comparing the effect of the propofol, etomidate and propofol + etomidate admixture on heart rate (Fig. 2) it was seen that the baseline values in all the three groups were comparable with no statistical difference among them $(p>0.05)$. There was no significant variation in heart rate at 1 min after the administration of drugs in all the three groups ( $p>0.05$ ), while the increase in heart rate at 1, 3, 5 and 10 mins after laryngoscopy and intubation was more in etomidate group as compared to propofol + etomidate and propofol group $(\mathrm{p}<0.05)$.

On comparing the effect of drugs on systolic, diastolic and mean arterial blood pressure (Fig. 3) between the three groups, it was seen that the baseline values in all the groups were comparable with no statistical difference among them ( $p>0.05$ ). But $1 \mathrm{~min}$ after induction it was observed that there was no effect on systolic, diastolic and mean blood pressure in patients receiving etomidate and etomidate+propofol, whereas patients who received propofol had significant fall $(\mathrm{p}<0.05)$ in blood pressure. Similarly, after laryngoscopy and intubation it was seen that there was a stable and significant rise in systolic, diastolic and mean blood pressure with etomidate and propofol + etomidate.

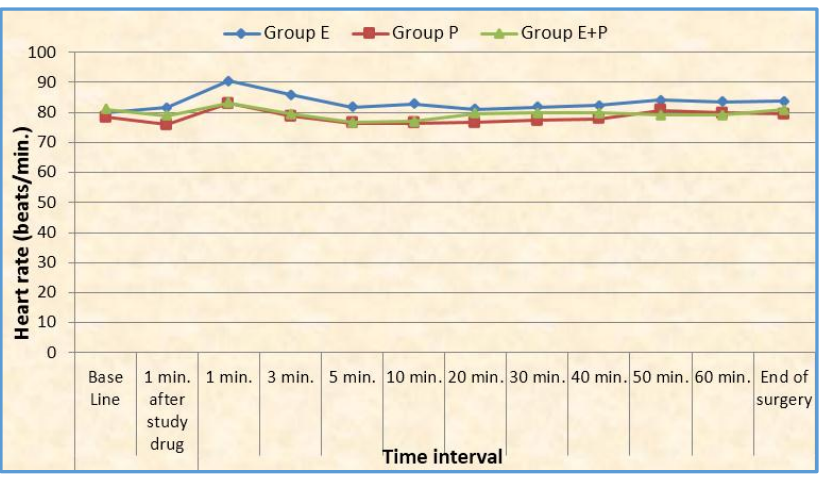

Figure 2. Heart Rate

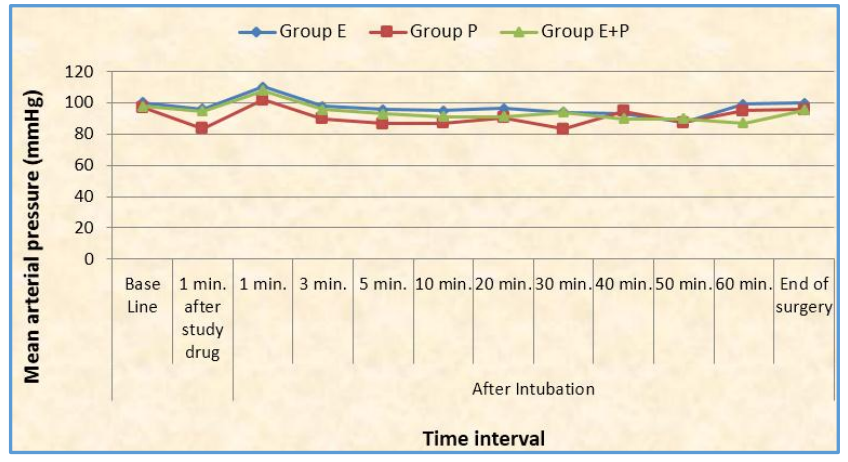

Figure 3. $M B P$

On comparing postoperative nausea and vomiting, the incidence of postoperative nausea was decreased to a highly significant level $(\mathrm{p}<0.0001)$ after the propofol + etomidate $(23.34 \%)$, while the incidence was maximum in group of patients who received etomidate $(56.67 \%)$ and least with propofol $(16.67 \%)$ among all the three groups. Similarly, the incidence of vomiting was high in patients who received etomidate $(16.67 \%)$, whereas no incidence of vomiting was reported in patients who received propofol or propofol+etomidate.

\section{DISCUSSION}

Our study was conceptualised to compare the induction characteristics and postoperative nausea and vomiting with propofol, etomidate lipuro and coinduction with propofol+etomidate lipuro.

The term Coinduction was introduced in 1986 and was applied to the use of two or more drugs for induction of anaesthesia. Coinduction of anaesthesia is a technique which involves drug interactions, particularly synergism, principally between two or more drugs and improves all the phases of anaesthesia including induction, maintenance and recovery.(4)

The use of etomidate lipuro and propofol combination in the ratio of 1: 1 can be used for induction in general anaesthesia, as these two drugs were studied for availability of admixture and it was reported that these drugs can be mixed and are physically compatible.(5)

In our study, we observed that pain on injection was least with the use of propofol-etomidate-lipuro mixture $(p<0.0001)$ as compared to either drug used alone and was 
maximum with the use of propofol. The use of propofol has been associated with high incidence of pain on injection. Etomidate-lipuro contains etomidate dissolved in a fat emulsion of medium and long chain triglycerides having almost physiological osmolality and very less incidence of pain on injection.(6) So coinduction with a mixture of propofol and etomidate lipuro decreases the incidence of pain on injection due to decreased concentration of propofol and because of presence of medium and long chain triglycerides in etomidate lipuro. This is in concordance with earlier study done by Saricaoglu et al, who reported that etomidate and propofol admixture resulted in lesser incidence of pain on injection. ${ }^{(5)}$

In our study, we observed that the increase in heart rate after laryngoscopy and intubation was more in etomidate group as compared to other two groups, but it was statistically insignificant ( $p>0.05$ ) and returned to baseline values after 10 minutes. Etomidate does not blunt the haemodynamic response to laryngoscopy and tracheal intubation, therefore brief episodes of increase in heart rate and blood pressure may be observed which may however not contribute to adverse outcome.

Results of our study showed that on comparing mean blood pressure between the three groups, Propofol group was associated with significant fall in blood pressure as compared to other two groups. Propofol induced hypotension during induction is mediated by inhibition of the sympathetic nervous system and impairment of the baroreflex regulatory mechanism. Propofol may lead to a reduction in the systemic vascular resistance and cardiac output. Also propofol is considered to have a direct relaxant effect on venous smooth muscles and in this way an increase in venous capacitance may contribute to the hypotension in patients.(7) On another side, haemodynamic stability observed with etomidate may be due to its unique lack of effect on the sympathetic nervous system and on baroreceptor functions.(7),(8),(9),(10) The decreased concentration of propofol and the haemodynamic stable effect of etomidate might have reduced the incidence of fall in blood pressure after induction and maintained the haemodynamic stability after intubation and intraoperatively with propofol + etomidate coinduction.

Published literature on propofol shows that it possesses significant antiemetic activity and has been used successfully to treat postoperative nausea.(11,12) This antiemetic effect may be due to its serotonin antagonist effect or decreased synaptic transmission in the olfactory cortex causing a decrease in the release of excitatory amino acids such as glutamate and aspartate.(12) In our study, the reduced incidence of postoperative nausea and vomiting associated with propofol + etomidate group as compared to etomidate group can be due to the use of reduced dose of etomidate $(0.2 \mathrm{mg} / \mathrm{kg})$ and the antiemetic effect of propofol.

We have not observed the incidence of myoclonus in our study, but review of literature shows that is a common problem experienced during induction with etomidate. Etomidate causes depression of the cortex, which leads to transient disinhibition of the subcortical structure. Myoclonic movements can be a problem in full stomach patients, because of the risk of regurgitation and aspiration.

We did not measure the cortisol levels of the groups, but a well-documented side effect of etomidate is suppression of the adrenal synthesis of cortisol.[13,14]

\section{CONCLUSION}

We concluded from our study that coinduction with propofol and etomidate is associated with least incidence of pain on injection and more stable haemodynamics after induction, intubation and during intraoperative period. Combination of etomidate with propofol would decrease the required dose of both the medications, thereby decreasing the incidence of adverse effects associated with individual drugs. The incidence of postoperative nausea and vomiting was also decreased with the combined admixture of both the drugs. Hence, the combination of propofol + etomidate is a valuable agent for induction of anaesthesia.

\section{REFERENCES}

[1] Schaub E, Kern C, Landau R. Pain on injection: a double-blind comparison of propofol with lidocaine pretreatment versus propofol formulated with longand medium-chain triglycerides. Anesth Analg 2004;99(6):1699-702.

[2] Hosseinzadeh $\mathrm{H}$, Eidy M, Golzari SE, et al. Hemodynamic stability during induction of anesthesia in elderly patients: Propofol + Ketamine versus Propofol + Etomidate. J Cardiovasc Thorac Res 2013;5(2):51-4.

[3] Mayer M, Doenicke A, Nebauer AE, et al. Propofol and etomidate-Lipuro for induction of general anesthesia. Hemodynamics, vascular compatibility, subjective findings and postoperative nausea. Anaesthesist 1996;45(11):1082-4.

[4] Kam E, Abdul-Latif MS, McCluskey A. Comparison of Propofol-Lipuro with propofol mixed with lidocaine 10 mg on propofol injection pain. Anaesthesia 2004;59(12):1167-9.

[5] Saricaoglu F, Uzun S, Arun O, et al. A clinical comparison of etomidate-lipuro, propofol and admixture at induction. Saudi J Anaesth 2011;5(1):626.

[6] Nyman Y, Von Hofsten K, Palm C, et al. EtomidateLipuro is associated with considerably less injection pain in children compared with propofol with added lidocaine. Br J Anaesth 2006;97(4):536-9.

[7] Ebert TJ, Muzi M, Berens R, et al. Sympathetic responses to induction of anesthesia in humans with propofol or etomidate. Anesthesiology 1992;76(5):725-33.

[8] Aggarwal S, Goyal VK, Chaturvedi SK, et al. A comparative study between propofol and etomidate in patients under general anesthesia. Brazilian Journal of Anesthesiology 2016;66(3):237-41.

[9] Soliman HF. Bispectral index-guided induction of anesthesia by ketofol infusion provides the same cardiovascular stability like that of etomidate infusion. Ains Shams Journal of Anaesthesiol 2015;8(3):341-8.

[10] Shah SB, Chowdhury I, Bhargava AK, et al. Comparison of hemodynamic effects of intravenous etomidate versus propofol during induction and intubation using entropy guided hypnosis levels. J Anaesthesiol Clin Pharmacol 2015;31(2):180-5. 
[11] Arslan M, Çiçek R, Kalender HU, et al. Preventing postoperative nausea and vomiting after laparoscopic cholecystectomy: a prospective, randomized, doubleblind study. Curr Ther Res Clin Exp 2011;72(1):1-12.

[12] Eu-Gene K, Park HJ, Hyoseok K, et al. Antiemetic effect of propofol administered at the end of surgery in laproscopic assisted vaginal hysterectomy. Korean J Anesthesiol 2014;66(3):210-15.
[13] Wagner RL, White PF, Kan PB, et al. Inhibition of adrenal steroidogenesis by the anesthetic etomidate. $\mathrm{N}$ Engl J Med 1984;310(22):1415-21.

[14] Schenarts CL, Burton JH, Riker RR. Adrenocortical dysfunction following etomidate induction in emergency department patients. Acad Emerg Med 2001;8(1):1-7. 